\title{
Analysis and verification of multi-rotors attitude control algorithms in
}

\section{Pixhawk}

\author{
Fangzhen $\operatorname{Lin}^{1}$, a \\ 1Beihang University, China
}

Key words.Pixhawk, attitude control, angle error, cascade PID

\begin{abstract}
Pixhawk is an open-source flight control system. The research on the attitude control algorithm Pixhawk uses provides a good reference for the use and development of the Pixhawk. In this paper, it provides a theoretical analysis of the attitude control algorithm of multi-rotors in Pixhawk, and it also carried out simulations and flight experiments to verify the performance of the multi-rotor attitude control algorithm. It has shown that the attitude angle error algorithm based on rotation vector and cascade PID controller the Pixhawk uses not only can stabilize the multi-rotor around hovering, but also can realize complex maneuvers.
\end{abstract}

\section{introduction}

in recent years, the UAV is increasingly popular, especially small scale UAV, represented by the multi-rotor. Flight control systems as the core of UAV also attract more and more attention, at the same time, open source flight control systems have received considerable attention from research communities. with reliable hardware and excellent software algorithm, Pixhawk is most popular open source flight control system, while the research on the control algorithm in Pixhawk is poor, in this paper, the attitude control algorithm of multi-rotors is concerned, we mainly talk about the method of calculating the angle error and attitude controller, at the same time, we conduct simulation experiments and flight experiments to verify the attitude control algorithm.

\section{The attitude control algorithm of multi-rotors}

The attitude angle error algorithm In Pixhawk, angle errors are calculated based on rotation vector while angle errors are subtraction between desired attitude angle and current attitude angles conventionally[1-3]. In this paper, we denote roll-pitch-yaw angles of the multi-rotor as $\phi, \theta, \psi$ and denote target roll-pitch-yaw angles as $\phi_{c}, \theta_{c}, \psi_{c}$,we represent their corresponding direction cosine matrix using $L_{g b}$ and $L_{g b^{c}}$, we also define the corresponding body frame using $O x_{b} y_{b} z_{b}$ and $O x_{b}^{c} y_{b}^{c} z_{b}^{c}$. as we know , the third row of the $L_{g b}$ is the projection of $o z_{b}$-axis in NED coordinate system, denoted as $z_{b} g$, similarly the third row of the $L_{g b^{c}}$ is the projection of $o z_{b}^{c}$-axis in NED coordinate system, denoted as $z_{b}^{c} g$.in addition, $z_{b} g$ and $z_{b}^{c} g$ are unit vector, the dot product of $z_{b} g$ and $z_{b}^{c} g$ is the cosine of 
the angle between $z_{b} g$ and $z_{b}^{c} g$, due to the monotonic of the cosine function between we can use the angle between $[-\pi \pi]$, so we can use $z_{b} g z_{b}^{c} g$ to measure the deviation between the current attitude and target attitude. We use the different method to calculate the angle error between the current attitude and target attitude according to the value of $z_{b} g z_{b}^{c} g$.

if $z_{b} g z_{b}^{c} g>0$, we consider that the deviation between the current attitude and target attitude is small, angle errors are calculated by the following method:

1) Rotating the multi-rotor around the axis of rotation to make the $o z_{b}$-axis coincide with $o z_{b}^{c}$-axis, as the figure 1 shows.
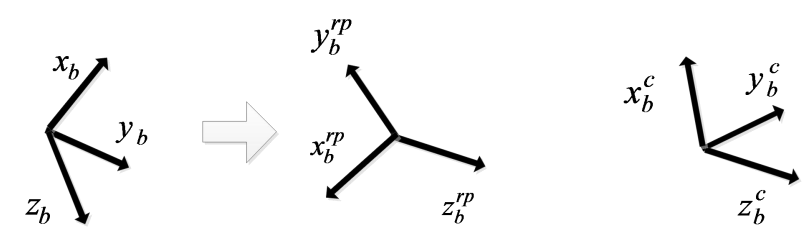

Figure 1

We denoted the axis of rotation and the angle of rotation as $e_{-} R_{-} z_{-}$axis and $e_{-} R_{-} z_{-}$angle.$e_{-} R_{-} z_{-}$axis is the axis of rotation expressed in current body frame $O x_{b} y_{b} z_{b}$, and the $e_{-} R$ is the vector which consists of rotation angles around the axis of $O x_{b}^{c} y_{b}^{c} z_{b}^{c}$ in that process. As the formula (1), (2), (3) shows blew.

$$
\begin{aligned}
& e_{-} R_{-} z_{-} \text {axis }=\{l, m, n\}^{T}=L_{g b}{ }^{T} \frac{z_{b} g \times z_{b}^{c} g}{\left\|z_{b} g \times z_{b}^{c} g\right\|} . \\
& e_{-} R_{-} z_{-} \text {angle }=\arctan \left(\frac{z_{b} \times z_{b}^{c}}{z_{b} z_{b}^{c}}\right) . \\
& e_{-} R^{\prime}=e_{-} R_{-} z_{-} \text {axis } \times e_{-} R_{-}{ }^{z} \text { angle. }
\end{aligned}
$$

After the rotation above, the multi-rotor reach a transition attitude, the corresponding body frame and direction cosine matrix are denoted as $O x_{b}^{r p} y_{b}^{r p} z_{b}^{r p}$ and $L_{g b}^{r p}$. As the formula (4), (5) shows blew.

$$
L_{g b}^{r p}=L_{g b} \times\left(\mathrm{I}+U \sin \theta+(1-\cos \theta) U^{2}\right) .
$$




$$
U=\left(\begin{array}{ccc}
0 & -n & m \\
n & 0 & -l \\
-m & l & 0
\end{array}\right)
$$

2) Rotating the multi-rotor around $o z_{b}^{c}$-axis to make the $O x_{b}^{r p} y_{b}^{r p} z_{b}^{r p}$ coincide with the target body frame $O x_{b}^{c} y_{b}^{c} z_{b}^{c}$, as figure 2 shows.

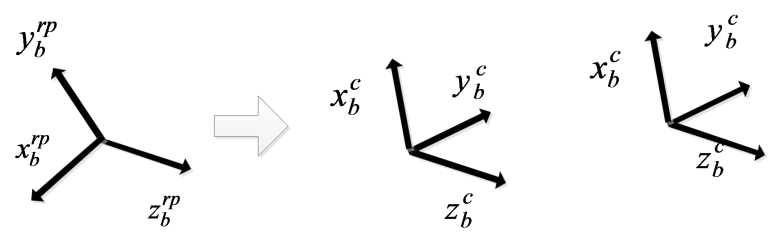

Figure 2

The rotation angle is denoted as $y a w_{-} e r r$.the first row of direction cosine matrix $L_{g b}^{r p}$ is $o x_{b}^{r p}$-axis expressed in NED coordinate system, denoted as $x_{b}^{r p} \_g$, similarly, the first row of direction cosine matrix $L_{g b}^{c}$ is $o x_{b}^{c}$-axis expressed in NED coordinate system, denoted as $x_{b}^{c} g, x_{b}^{r p}{ }_{-} g$ and $x_{b}^{c} g$ are unit vectors we can get the $y a w_{-} e r r_{\text {from }}$ them. As the formula (6) shows blew.

$$
y a w_{-} \text {err }=\arctan \left(\frac{\left(x_{b}^{r p}-g \times x_{b}^{c}-g\right) \times z_{b}^{c} g}{x_{b}^{r p}{ }_{-} g \bullet x_{b}^{c} g_{-}}\right) .
$$

3) Calculate the angle error

During the flight, pilots mainly adjust the roll angle and pitch angle, so in the first rotation process, $e_{-} R(3)$ is smaller than $e_{-} R(1)$ and $e_{-} R(2)$ that can be ignored. Pitch and roll controller is mainly doing tracking controller. In the second rotation process, yaw controller is mainly doing tracking controller. As we know, the response of the motion of pitch and roll is faster than yaw. The decomposition decouples the multi-rotor to sequentially execute fast response motion and slow response motion.

So in the whole process, to make current the body frame coincide with target body frame, the rotation angle around $O x_{b}$-axis is $e_{-} R(1)$, the rotation angle around $O y_{b}$-axis is $e_{-} R(2)$, to eliminate the influence of the slow response of yaw motion, the rotation angle around $O z_{b}$-axis can be $y a w_{-} e r{ }^{*} \cos \phi^{c 2} \cos \theta^{c 2}$, if the target attitude is near hovering, the $y a w_{-} e r r^{*} \cos \phi^{c 2} \cos \theta^{c 2}$ is bigger that make it easier to return hovering for multi-rotors.

Overall, if $z_{b} g z_{b}^{c} g>0$, angle errors of roll-pitch-yaw angles are $e_{-} R(1) 、 e_{-} R(2)$ 、 $y a w_{-} e r r^{*} \cos \phi^{c 2} \cos \theta^{c 2}$. 
if $z_{b} g z_{b}^{c} g<0$, we consider that the deviation between the current attitude and target attitude is large, angle errors are calcaulated by the following method:

1) Rotating the multi-rotor around the axis of rotation to make the $O x_{b} y_{b} z_{b}$ coincide with $O x_{b}^{c} y_{b}^{c} z_{b}^{c}$, as figure 3 shows.
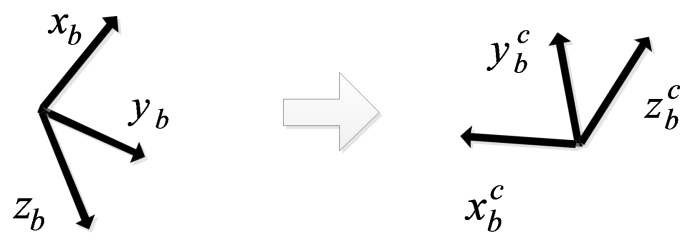

Figure 3

The direction cosine matrix of rotation process can be represented by $C_{b b^{\prime}}, C_{b b^{\prime}}$ can be expressed with a group of quaternion, the quaternion is denoted as $q=\left\{q_{0}, q_{1}, q_{2}, q_{3}\right\}$. We also denote the rotation-axis as $e_{-} R_{-} d$ and the vector of rotation angles as $e_{-} R_{-} d_{-}$angle. As the formula (7), (8), (9) shows blew.

$$
\begin{aligned}
& C_{b b^{\prime}}=L_{g b}{ }^{T} L_{g b^{c}}=\left(\begin{array}{ccr}
q_{0}{ }^{2}+q_{1}^{2}-q_{2}{ }^{2}-q_{3}^{2} & 2\left(q_{1} q_{2}-q_{0} q_{3}\right) & 2\left(q_{0} q_{2}+q_{1} q_{3}\right) \\
2\left(q_{1} q_{2}+q_{0} q_{3}\right) & q_{0}^{2}-q_{1}^{2}+q_{2}{ }^{2}-q_{3}^{2} & 2\left(q_{2} q_{3}-q_{0} q_{1}\right) \\
2\left(q_{0} q_{2}-q_{1} q_{3}\right) & 2\left(q_{2} q_{3}+q_{0} q_{1}\right) & q_{0}{ }^{2}-q_{1}{ }^{2}-q_{2}{ }^{2}+q_{3}{ }^{2}
\end{array}\right) . \\
& e_{-} R_{-} d=\frac{\left\{q_{1}, q_{2}, q_{3}\right\}}{\left\|q_{1}^{2}+q_{2}^{2}+q_{3}^{2}\right\|} . \\
& e_{-} R_{-} d_{-} \text {angle }=e_{-} R_{-} d^{*} \arctan \left(\frac{q_{0}}{\sqrt{\left\|q_{1}^{2}+q_{2}^{2}+q_{3}^{2}\right\|}}\right) .
\end{aligned}
$$

2) Calculate the angle error

In the whole process, to make current the body frame coincide with target body frame, the rotation angle around $O x_{b}$-axis, $O y_{b}$-axis, $O z_{b}$-axis are

$$
e_{-} R_{-} d_{-} \text {angle(1), } e_{-} R_{-} d_{-} \text {angle(2), } e_{-} R_{-} d_{-} \text {angle(3). }
$$

In order to get continuous angle errors, the final angle error can be expressed with a weighted average of angle errors got when deviation is large and small. The final angle error is denoted as error . As the formula (10), (11) shows blew.

$$
\begin{aligned}
& \text { direct_w }=\left(z_{b} g z_{b-}^{c} g\right)^{2} * \cos \phi^{c 2} \cos \theta^{c 2} . \\
& \text { error }=\left\{\mathrm{e}_{-} \mathrm{R}(1), \mathrm{e}_{-} \mathrm{R}(2), y a w_{-} \text {err } * \cos \phi^{c 2} \cos \theta^{c 2}\right\}(1-\text { direct_w })+\mathrm{e}_{-} \mathrm{R} \_\mathrm{d} \cdot \text { direct_w. }
\end{aligned}
$$


direct ${ }^{w}$ will get bigger when the angle deviation is bigger, so the weight of angle errors got when angle deviation is bigger. In addition, if the target attitude around hovering, the angle error calculated is bigger, so feedback is bigger, multi-rotor can return hovering more quickly.

At the same time, with the angle error calculated by rotation vector, the path of attitude regulation is shorter than conventional algorithms[4-7].

Cascade PID attitude controller

In Pixhawk, the attitude controller is the cascade PID controller, the inner loop is to control the attitude angular rate which responds quickly, the outer loop is to control the attitude angle which responds slow. As figure 4 shows.

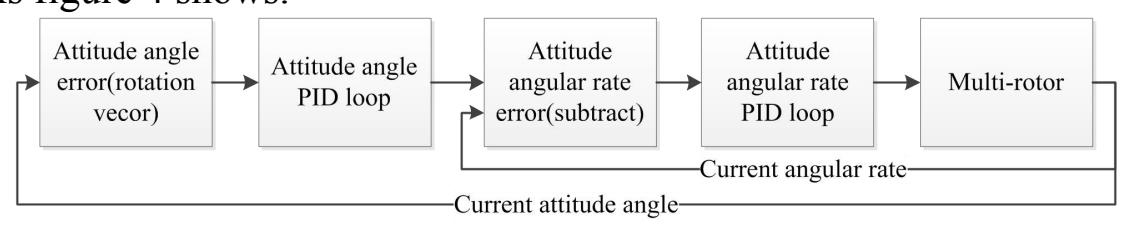

Figure 4

Firstly, getting the angle error using the method shown above when the current attitude and target attitude is known, secondly, getting the target angular rate from the outer PID controller, thirdly, target angular rates subtract current angular rates to get angular rates. Lastly, getting the final output from the inner PID controller. Overall, Multi-rotors can follow the target attitude angular rate, and thus can be able to follow the target attitude angle[8-10].

\section{Verification of the effect of the controller}

In order to verify the effect of the control algorithm, we establish flight control system mathematical model of multi-rotors and conduct simulation experiments, on the other hand, we conduct flight experiments using a four-rotor aircraft which uses the control algorithm show above. The simulation experiment

The performance of the control algorithm can be compared by step response. In simulation experiments, several typical cases are validated[11].

1) Multi-rotor is hovering initially, a step response is generated to simulate a control command that make pitch angle and roll angle reach 5 degrees. As figure 5 shows

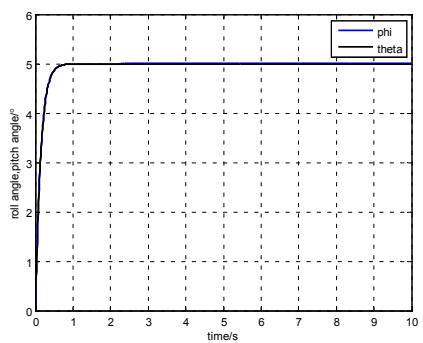

Figure 5

2) Multi-rotor is hovering initially, a step response is generated to simulate a control command that make pitch angle and roll angle reach 50 degrees. As figure 6 shows 


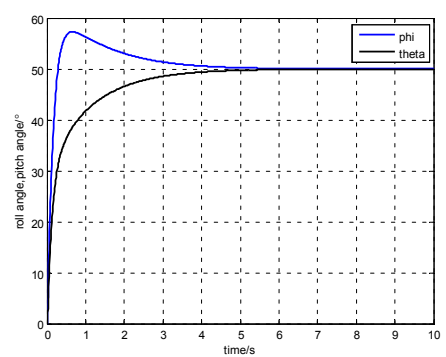

Figure 6

\section{Flight experiment}

We also carry out flight experiments outdoor. Figure 7 shows the control performance during flight. The error between the target attitude and current attitude is less than $3^{\circ}$ conventionlly, even doing large angle movement, it can also track the target attitude quickly and precisely. As figure 7 shows

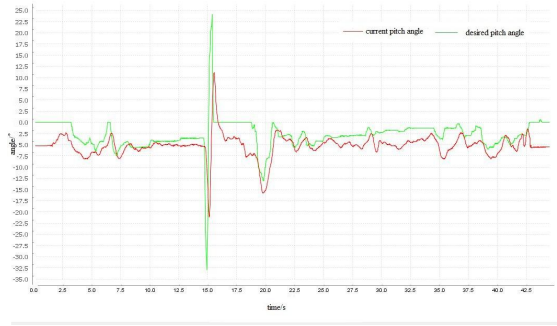

Figure 7

From the result shown above, we can know that multi-rotor can control the attitude quickly and precisely with the control algorithm.

\section{Conclusion}

In this paper, we present the angle errors which calculated based on rotation vector and cascade PID controller based on the attitude angle and the angular rate. With the algorithm, multi-rotors can make the path shorter and smoother when changing the attitude, and track the target attitude quickly and precisely which is important for flight control systems.

\section{References}

[1]. Jiaji He, Modeling the Quad-rotor and Control Strategy research[D]. Northeastern University. 2012. In Chinese.

[2]. Guangchao Tan, Design and Implementation of Attitude Control System for a Quadrotor UAV. Dalian University of Technology. 2013. In Chinese.

[3]. Lei Zhang, Hao Li, Attitude Control of Four- Rotor Aircraft via Fuzzy PID. Computer Simulation, 2014(08): p.73-77. In Chinese.

[4].Yun, Y., et al. High performance full attitude control of a quadrotor on SO(3). in IEEE International Conference on Robotics and Automation. 2015.

[5].Mellinger, D. and V. Kumar, Minimum snap trajectory generation and control for quadrotors. 2011 IEEE International Conference on Robotics and Automation.2011.

[6]. Mellinger, Daniel Warren, Trajectory Generation and Control for Quadrotors. Publicly Accessible Penn Dissertations.2012, p.524-547. 
[7].McKerrow, P.,Modelling the Draganflyer four-rotor helicopter. IEEE International Conference on Robotics \&amp. 2004.

[8]Qingrui Feng, Hailong Pei, The application of cascade PID control in UAV attitude control. Microcomputer Information, 2009. 25(22): p.9-10. In Chinese.

[9].Chengfu $\mathrm{Wu}$, Xiaoqi Liu, Xu Yuan, Modeling and PID control for a quadrotor. Electronic Design Engineering, 2012(16): p.68-70. In Chinese.

[10]. Xiaobo Li, Shuxi Song, Hovering Control for Quadrotor Unmanned Helicopter Based on Fuzzy Self-tuning PID Algorithm. Control Engineering of China, 2013(05): p.910-914. In Chinese.

[11]. Shaobin Ding, Modeling and Quaternion Control of X-Type Quadrotor. Journal of System Simulation, 2015(12): p.57-62. In Chinese. 